\title{
Clusters Deposition on Surface an Atomic Scale Study by Computer Simulation Method
}

\author{
Akbarali Rasulov', Nodirbek Ibroximov² \\ ${ }^{1}$ Ferghana Branch of Tashkent University of Information Technologies, Ferghana, Uzbekistan \\ ${ }^{2}$ Ferghana Polytechnical Institute, Ferghana, Uzbekistan \\ Email: arasulov59@mail.ru,n_ibrohimov@mail.ru
}

How to cite this paper: Rasulov, A. and Ibroximov, N. (2019) Clusters Deposition on Surface an Atomic Scale Study by Computer Simulation Method. Journal of Applied Mathematics and Physics, 7, 2303-2314.

https://doi.org/10.4236/jamp.2019.710156

Received: July 29, 2019

Accepted: October 11, 2019

Published: October 14, 2019

Copyright (C) 2019 by author(s) and Scientific Research Publishing Inc. This work is licensed under the Creative Commons Attribution International License (CC BY 4.0).

http://creativecommons.org/licenses/by/4.0/

\begin{abstract}
The investigation is generalized to clusters with sizes up to 3000 atoms, covering this way the range of sizes experimentally available for low energy cluster beam deposition. The atomic scale modeling is carried on by both Molecular Dynamics and Metropolis Monte Carlo. This represents a huge series of simulations (175 cases) to which further calculations are added by spot when finer tuning of the parameters is necessary. Analyzing the results is a major task which is still in progress. This way, not only a realistic range of sizes is covered, but also the whole range of compositions and the temperature range relevant to the solid and the liquid states.
\end{abstract}

\section{Keywords}

Computer Simulation, Low Energy, Cluster, Deposition, Slowing down, Molecular Dynamics, Parallelization, Embedded Atom Model

\section{Introduction}

Nowadays, computer simulations play a very important role in research. In the past, research was characterized by the interplay between theory and experiment. With the advent of high speed computers (started to be utilized since the $50 \mathrm{~s}$ ), this picture changed; computer simulation methods (or so-called "computer experiments") became important in research due to their ability to "bridge" theory and experiment. One of the advantages of the computer experiments is that process parameter interdependencies can be resolved, which might be difficult or impossible to do in a real experiment. Moreover, computer simulations can often be performed for conditions close to the experimental conditions and the obtained simulation results can be compared directly with experimental observations. Other simulations may not correspond to experimental 
conditions, but still may provide complementary information. This, in turn, makes computer simulations an extremely powerful tool to both understand phenomena underlying experimental observations, but also to study regions that are not attainable experimentally. A report is presented about progress in the understanding of the properties of bi-metallic nanoparticles, their interaction with surfaces subsequent to low energy slowing down and the properties of nanostructured materials formed with these particles. A nanoparticle contains from a few atoms for the smallest ones to several thousand for the largest ones considered here. The properties of an atom result from quantization and the same is true for the molecules they form. The same is thus true for the smallest nanoparticles. At the other edge, many of the properties of macroscopic materials are well described by a classical approach and nanoparticles appear as objects at the fringing field between quantum and classical behaviors. In the study of their properties, using either a quantum or a classical approach, atomic scale methods appear as naturally well-suited. Atoms are considered as individual objects interacting via their outer shell electrons only. However, even with such an approximation, solving the Schrödinger equation becomes quickly prohibitively heavy as the number of atoms involved increases. For the heaviest elements, relativistic effects make the problem even heavier. In this case, the classical approach is the only one presently practical.

Of particular interest are the conditions to fulfill in order to get core-sell structures, the factors governing the clusters morphology, those governing the short and long range order in the clusters as a whole as well as of the silver and cobalt subsystems, the conditions for melting as well as the order of the solid-liquid phase transition in the clusters, and the conditions for Co dissolution in the Ag-Co clusters. A comprehensive study is in progress which aims at capturing all the possible thermodynamic states of Co-Ag clusters and the transitions from one state to another. Phenomena about which understanding may already be put forward are the energetic and stoechiometric conditions for the precipitation of clusters into a core-shell structure, the wetting of cobalt cores by silver in clusters containing mainly cobalt, the stoechiometric conditions for a roughening transition of spherical clusters (and vice-versa), the disordering of silver shells, the role of the cluster surface and of the internal $\mathrm{Co} / \mathrm{Ag}$ interface in the dynamics of melting, the occurrence of a solid-liquid coexistence temperature and the condition for Co dissolution in clusters containing mainly silver.

\section{MD Simulations}

Owing to fast and powerful computers large and complex systems can be investigated using computer simulations. To main families of simulation techniques at the atomic scale are Monte-Carlo and Molecular Dynamics simulations; a variety of hybrid models that combine features from both techniques exist as well. The MD model employed is already described elsewhere [1] [2] [3] [4] and will only be briefly summarized. The equations of motion of the atoms in the system 
are integrated stepwise in time with the algorithm in [5]. Forces are derived from a Embedded Atom Model potential (EAM) proposed in [1] and account, in addition, for a contribution of electron-phonon coupling. This is done by means of a friction term which governs the exchange of energy between the ionic and the electronic systems, assuming a constant electronic temperature. It is shown in [6] how an approximate model can be established to evaluate the strength of the coupling with no adjustable parameters. The physical quantities needed are known from experiment in the case of pure elements, and it is assumed that the electronic density at the Fermi level is one electron per atom. The electron-phonon coupling contributes to dissipate the energy brought by the cluster in the impact and enhances the local cooling of the system. As compared to elemental systems, more complexity occurs in the present case as we have to deal with two different metallic elements that are not homogeneously distributed. The approximated electron-phonon coupling model employed is unsuitable to correctly describe the transport of heat by the electronic system through an interface between two elemental subsystems as in a core-shell structured cluster. It is considered here that, since the substrate is pure silver and to neglect the difference with cobalt. The major parameter which governs the interatomic interactions in the system is, of course, the potential.

Its assessment for the Co-Ag system is thoroughly discussed in [4] [6] and this discussion will not be repeated. The shortest Ag-Ag separation distance involved in the simulations presented below is $2.124 \AA$ at $1.5 \mathrm{eV} /$ at. According to Figure 1 , separation distances with the EAM potential energy equal to $1.5 \mathrm{eV}$ is $2.47 \AA$, and $2.21 \AA$ with the Molière potential energy equal to $1.5 \mathrm{eV}$. These distances are significantly closer to the range of applicability of the EAM model than to the range of applicability of screened Coulomb potentials. The discrepancy between screened Coulomb and EAM forces is the largest for the Ag-Ag system and the discussion above is also valid for Co-Co and Ag-Co interactions.

This potential was used to discuss the equilibrium properties of Co clusters embedded in Ag and Co-Ag free clusters. The difference with the present case is the impact of the clusters on a surface, involving energies up to $1.5 \mathrm{eV}$ per atom, which is much higher than those involved at thermal equilibrium. However, at this energy, the shortest $\mathrm{Ag}-\mathrm{Ag}$ separation distance involved in the simulations presented below is $2.124 \AA$ at $1.5 \mathrm{eV} /$ at. It is similar for Co-Co and Ag-Co pairs at the same energy. Such distances are still of the order of the first neighbor distance for which the EAM potential is designed. In order to evaluate the modification of the clusters as a result of their impact on an Ag substrate surface, a set of characterization functions is used.

A structure factor is used to measure the epitaxial accommodation of the clusters with the substrate. It is measured inside the cluster and gives information about the periodicity in one direction (1).

$$
S=\frac{1}{N} \sum_{j=1}^{N} \mathrm{e}^{i k r_{j}}
$$




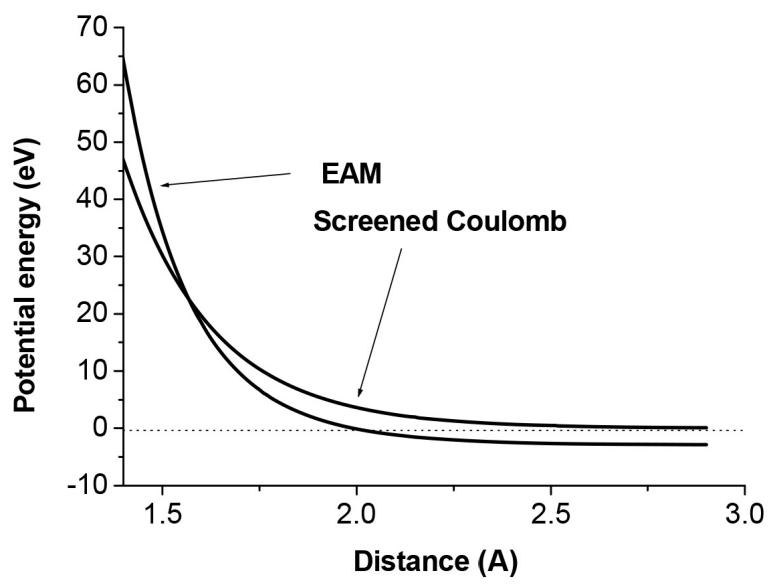

(a)

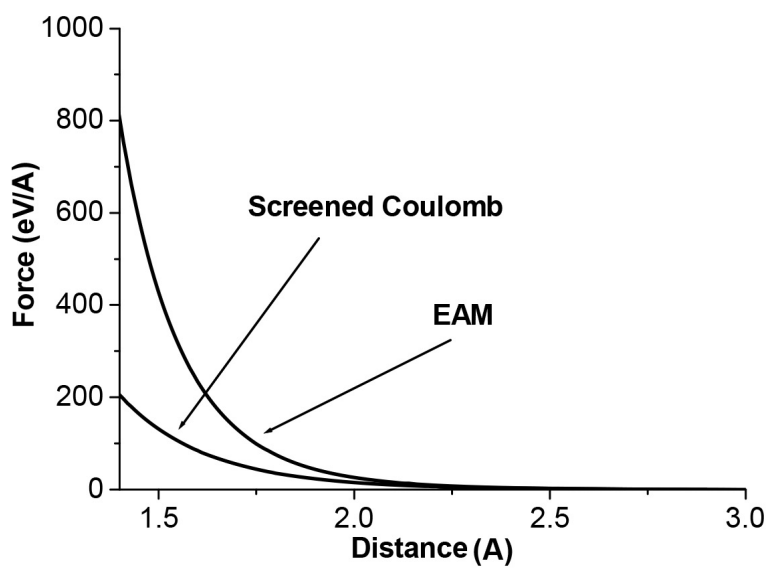

(b)

Figure 1. Comparison between the potential energies (a) and forces (b) as functions of the separation distance, between two atoms interacting via a screened Coulomb potential and via an EAM potential. In this latter case, the configuration energy of one atom is represented as a function of its distance to one of its first neighbor, its environment being kept constant.

In this expression, $k$ is the wave vector, $r_{j}$ is the position of the atom $j$ and $N$ is the total number of atoms in the cluster. If the periodicity in the direction of $\mathrm{k}$ corresponds to the inverse of then the value of is unity. If there is no such periodicity in this direction is zero. In order to measure the epitaxial accommodation of the deposited cluster with the substrate, substrate lattice wave vectors are used:

$$
\boldsymbol{k}=\frac{4 \pi}{a_{0}}(h, k, l)
$$

where $a_{0}$ is the substrate lattice parameter, and $h, k$ and $l$ are Miller indices of lattice directions. A pair correlation function is used to characterize short-range order in the clusters:

$$
g(r)=\frac{1}{2(N-1)} \sum_{i=1}^{N-1} \sum_{j=i+1}^{N} \delta\left(r_{i j}-r\right)
$$

where is the Dirac function, $N$ is the number of atoms in the cluster and $r_{i j}$ the distance between atoms $i$ and $j$ in the cluster. The pair correlation function gives the number of atomic pairs separated by a given distance, $r$. This function is calculated separately for the different kinds of pairs: $\mathrm{Co}-\mathrm{Co}, \mathrm{Ag}-\mathrm{Ag}$ and Ag-Co. It is characteristic of the lattice structure.

\section{Thin Film Growth by Low Energy Cluster Deposition}

Using Parallel Programming with MPI the $\mathrm{Ag}_{\mathrm{n}} \mathrm{Co}_{\mathrm{m}}$ clusters with $\mathrm{n}=\mathrm{m}$ (where $\mathrm{n}$ $=100,250,500,750,1000,1250$ and 1500) have been deposited on an Ag (100) surface at energies of $0.5 \mathrm{eV}$ per atom for the studying the thin film growth processes by LECBD. In this case the $\mathrm{Ag}_{\mathrm{n}} \mathrm{Co}_{\mathrm{m}}$ clusters with number of atoms 200, $500,1000,1500,2000,2500$ and 3000 are deposited consequently with randomize choosing the next cluster from mentioned list of clusters. The slowing down 
of each cluster is followed for $150 \mathrm{ps}$ and then next one. The substrate has a size $148.2 \AA \times 148.2 \AA \times 98.8 \AA$ which consists of 124,416 atoms. The calculation has been performed at room temperature with taking into account the periodic boundary condition on two dimensions and the electron-phonon coupling [7] [8].

The first characteristic of the slowing down in the energy range considered is the penetration of the clusters into the substrate and the related damage. Surface damage production induced by the soft landing of Co atoms was already studied, both experimentally and by MD in [1] and surface tunneling microscopy showed it to be significant. The situation may be different if the incident Co atoms are bound inside clusters as in the present case.

At the low energies considered (no more than $1.5 \mathrm{eV}$ per atom), the cluster penetration, if any, is only partial. Co is always found regrouped into one cluster surrounded by a layer of silver. When it penetrates, the cluster displaces Ag atoms from the substrate with the consequence of the formation of add-atoms. For both clusters, the fraction of cluster atoms with final positions below the substrate surface is the same and is close to linearly increasing with the slowing down energy. It is close to 3 percent at $0.25 \mathrm{eV} /$ at and 30 percent at $1.5 \mathrm{eV} /$ at. According to a rule of volume conservation, in the case of the smallest cluster, the number of substrate atoms displaced above the surface is exactly equal to the number of cluster atoms below this surface. It is somewhat smaller for the larger cluster since the volume per Co atoms below the surface is less than the volume per $\mathrm{Ag}$ atoms in the substrate.

Figure 2 shows the maximal penetration depth of the incident clusters (Figure 2(a)) and the height of the layer formed by substrate atoms above the surface (Figure 2(b)) as functions of the incident energy. The distinction is made between the penetration of each of the elements forming the incident clusters. The results are averaged over ten independent slowing downs at each energy and the standard errors on the mean are given. Not surprisingly, the maximal penetration is a monotonically increasing function of the incident energy and it is limited to 4 atomic layers for the largest cluster. The smallest cluster does not penetrate two layers, in the average, at the highest energy considered. The largest cluster systematically penetrates deeper than the smallest one. Systematically as well, Co, initially surrounded by Ag atoms has less penetration than Ag and still remains surrounded by its silver shell. Except for the smallest cluster with incident energy lower than $0.5 \mathrm{eV} / \mathrm{at}$, Co and $\mathrm{Ag}$ atoms are always found at least one layer deep in the substrate. This suggests that, in real experiments, substrate damage would be particularly difficult to avoid. As a consequence of the impact, Ag atoms leave the cluster and form add-atoms. Add-atoms are either found regrouped and forming a step at the periphery of the cluster, or they are found isolated at larger distances from the cluster. The latter, in their large majority, are found to originate from the cluster itself while the former are substrate atoms displaced during the penetration of the cluster. Part of the induced damage can be appreciated in Figure 2(b) where it is shown that 


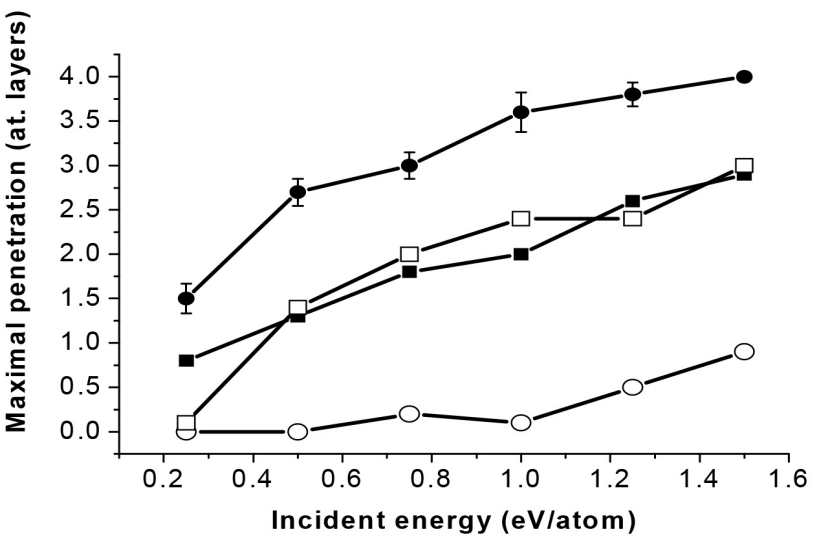

(a)

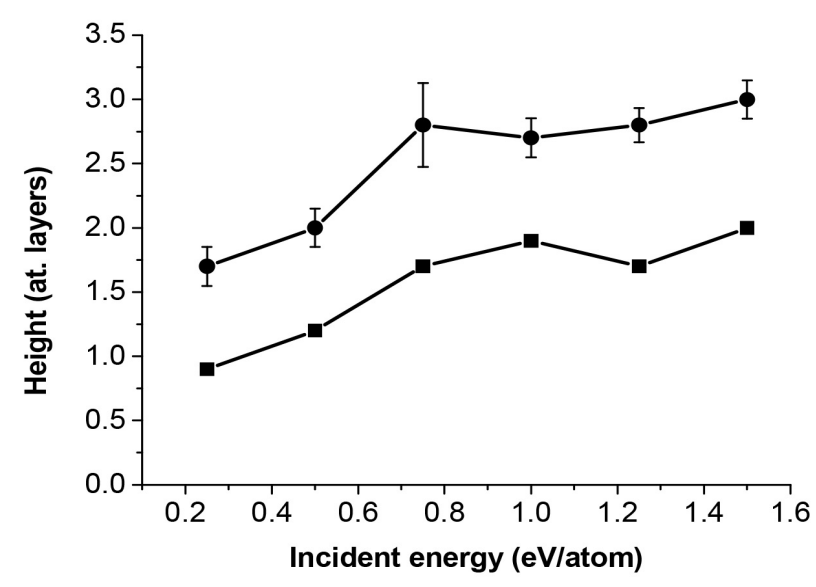

(b)

Figure 2. Maximal penetration (a) and mean height of the step around the clusters (b) as functions of the slowing down energy. In Figure 2(a), dark squares represent the results for Ag in the small cluster, dark circles for $\mathrm{Ag}$ in the big clusters, open circles for Co in the small clusters and open squares for Co in the big clusters. In Figure 2(b), circles provide the results for the small cluster, squares for the big clusters. The error bars displayed represent the standard error on the mean and are only shown for one curve per plot.

displaced $\mathrm{Ag}$ atoms form layers around the cluster which height, in the average, is also an increasing function of the incident energy. This means that a configuration with a compact two or three atomic layer step at the periphery of the cluster is energetically better favorable than a single monolayer configuration extending on a larger area.

Lattice distortions in the clusters result, at short range, in changes in atomic separation distance distributions. These are examined by means of pair correlation functions. Such correlation functions for Ag, Co and Ag-Co pairs are shown in Figure 3. They were obtained as time averages over the last 50 ps evolution of the large cluster impacting at $0.25 \mathrm{eV} /$ at. Figure 3 is representative of all cases investigated. The first neighbor peaks are well defined. Their positions, indicated in the figure, can be evaluated as functions of the slowing down energies and the results are given in Figure 3 for all cases considered. These positions are clearly independent of the incident energy and only slightly depend on the cluster size and the amount of Co in it. They are also similar to the positions of the first neighbor peak in the free clusters. Hence, the slowing down and the interaction with the substrate do not modify the mean first neighbor separations significantly. A close analysis of the pair correlation functions indicates that, except for Co-Co pairs, the first neighbor peaks are significantly asymmetrical. This asymmetry is a combined effect of anharmonicity in thermal vibrations and lattice distortions. The numbers in Table 1 illustrates the situation. This table compares full widths at half-maximum, noted $w$, of the first peak in the correlation functions for Ag-Ag, Co-Co and Ag-Co pairs. It also gives their half left and right half widths, noted $w_{l}$ and $w_{r}$ respectively, namely toward small and large separations. The results are given for the free $\mathrm{Co}_{285} \mathrm{Ag}_{301}$ cluster at $300 \mathrm{~K}$, the same cluster after its deposition at $0.25 \mathrm{eV} /$ atom and the same temperature, and 


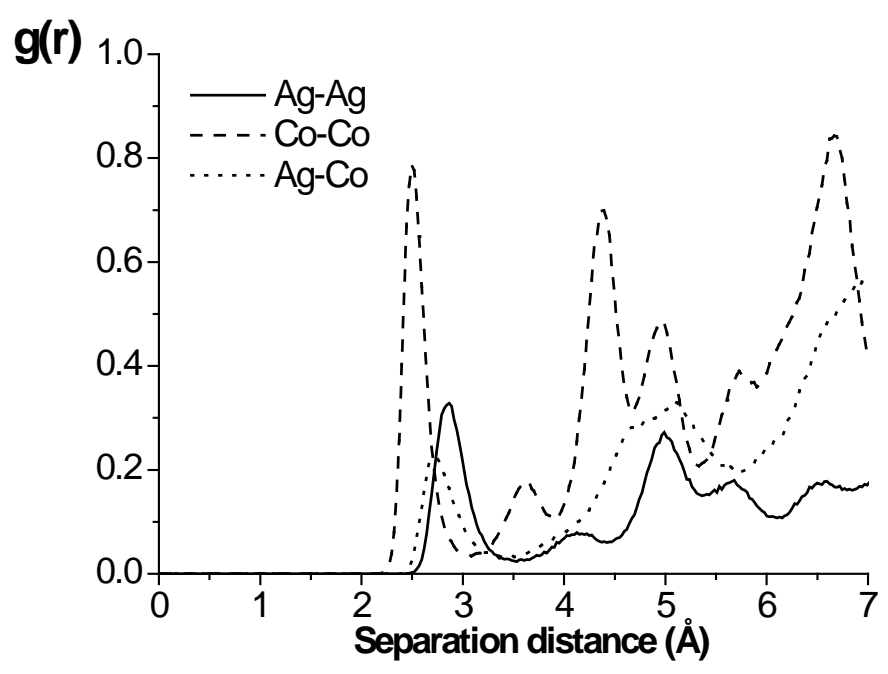

Figure 3. Pair correlation functions measured in the $\mathrm{Co}_{285} \mathrm{Ag}_{301}$ cluster after slowing down with $1.5 \mathrm{eV} /$ atom incident energy. Solid line: Ag-Ag pairs, dashed line: Co-Co pairs, dotted line: Ag-Co pairs.

Table 1. Characteristics of the first peak profile.

\begin{tabular}{ccccc}
\hline & & Ag-Ag & Co-Co & Ag-Co \\
\hline \multirow{3}{*}{ Free, 300 K } & w & 0.29 & 0.21 & 0.32 \\
& $\mathrm{w}_{\mathrm{l}}$ & 0.11 & 0.10 & 0.11 \\
& $\mathrm{w}_{\mathrm{r}}$ & 0.18 & 0.11 & 0.21 \\
& $\mathrm{w}$ & 0.34 & 0.23 & 0.33 \\
Deposited, 300 K & $\mathrm{w}_{\mathrm{l}}$ & 0.13 & 0.09 & 0.12 \\
& $\mathrm{w}_{\mathrm{r}}$ & 0.22 & 0.13 & 0.21 \\
Deposited, & $\mathrm{w}$ & 0.20 & 0.11 & 0.20 \\
OK & $\mathrm{wl}_{\mathrm{l}}$ & 0.05 & 0.07 & 0.04 \\
& $\mathrm{w}_{\mathrm{r}}$ & 0.16 & 0.04 & 0.16 \\
\hline
\end{tabular}

after quenching this deposited cluster and the substrate to $0 \mathrm{~K}$. The full widths are the largest for the deposited cluster at $300 \mathrm{~K}$.

In order to mimic a uniform collimated beam, the slowing down, always at normal incidence, is repeated ten times with the same cluster with a given initial kinetic energy, selecting the impact points on the surface and its orientations with respect to the surface at random. Each impact is followed during 150 ps at room temperature. Within the 150 ps evolution time considered, thermally stimulated configuration modifications may have a sufficiently high probability to take place. The slowing down is characterized by several significant features. These can be illustrated with the help of Figure 4 which represents a cut in the cluster before its deposition and in the cluster-substrate system after 150 ps evolution. The cluster $\mathrm{Co}_{285} \mathrm{Ag}_{301}$ is represented and the slowing down energy is 0.5 $\mathrm{eV} /$ atom.

The final system is characterized by a limited penetration of the cluster into the substrate. It undergoes some deformation accompanied by structural accommodation of the cluster with the substrate, which is limited in the case of 


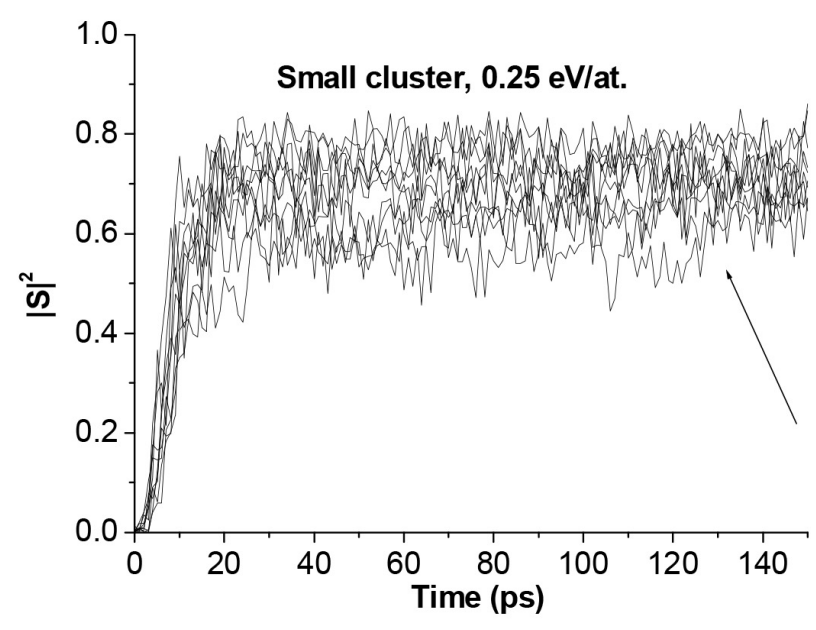

(a)

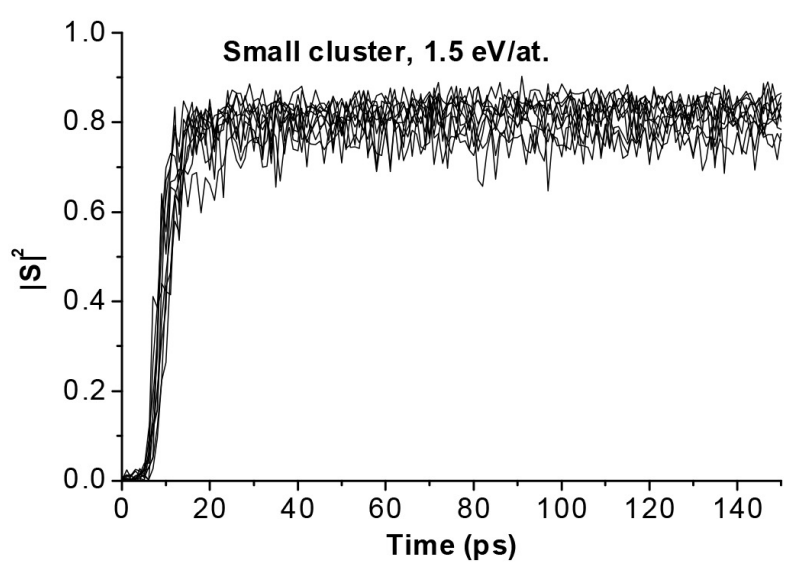

(b)

Figure 4. Structure factor as a function of time during the slowing down.

Figure 4. At the same time, the upper part of the cluster may retain its initial atomic arrangement. It the case of Figure 4, some damage is created in the substrate and the Ag cluster shell tends to spread on the substrate surface. While the Co core is only moderately affected by the slowing down, the Ag lattice, which is already distorted initially, undergoes further deformation as a consequence of the impact. All these characteristics of course depend on the incident energy as well as on the cluster size, composition and energy. We now turn to their systematic study for $\mathrm{CO}_{285} \mathrm{Ag}_{301}$ and $\mathrm{Co}_{10} \mathrm{Ag}_{191}$. Figure 4 provides information about the cluster accommodation evolution. In Figure 4(a), Figure 4(b) for most cases, the evolution is terminated after 30 ps. This is however not always the case, as pointed for one cluster in Figure 4(a) where a jump of the structure factor is identified, which is the signature a thermally activated process. Similar events were found in other cases not represented in Figure 4. The time of one impact, as defined above, is no longer than 5 ps.

The typical evolution time is close to one order of magnitude larger ( $30 \mathrm{ps}$ ) and, therefore, the cluster modifications cannot be assigned to the impact. This conclusion is supported by a detailed analysis of Figure 4(a), Figure 4(b) showing that the structure factor keeps close to zero during the time of the impact. It can be at least partially assigned to the local heating since the characteristic electron-phonon coupling time is 20 ps at room temperature in the present model. One thus has to conclude that the process of epitaxial accommodation starts after the impact is terminated and the combined effect of local heating and the substrate temperature is responsible for the epitaxial accommodation. This Figure 4 shows that, for the larger cluster, at low impact energy, the epitaxial accommodation is a still slower process. As shown by a regression line drawn for one of the clusters, the evolution is not always terminated after $150 \mathrm{ps}$. In this case, the impact energy ( $0.25 \mathrm{eV} /$ atom) produces less damage in the cluster than at $1.5 \mathrm{eV} /$ atom, so that the energy barrier to surmount for reaching epitaxial ac- 
commodation is higher. This energy barrier is probably to a large extent the energy required to rotate the Co group toward epitaxial correspondence with the substrate orientation.

If one defines the impact characteristic time as the time needed for the cluster to convert its center of mass kinetic energy into potential energy, and this potential energy to convert into kinetic energy into the whole system, it can be estimated as of the order of $5 \mathrm{ps}$, which is smaller than the electron-phonon coupling time at room temperature ( $20 \mathrm{ps}$ ). The slowing down of a cluster is followed during $150 \mathrm{ps}$ in order to track possible thermally activated processes. At the end of these 150 ps MD evolution, particle trajectories are fully decor related from the initial trajectories and the system is in a thermal equilibrium state which may be metastable. Whether this state has a sufficiently long lifetime to be observed is not known and this question needs comparison with experiment to be settled. However, if an incident cluster undergoes modifications because of the impact, the probability to retrieve its initial state once deposited is vanishingly small. These modifications may thus be considered as permanent, whatever further thermally activated modifications are still possible.

Each cluster impacts on the crystal surface at normal incidence with a given initial kinetic energy, selecting the impact points on the surface and its orientations with respect to the surface at random. Each impact is followed during 150 ps at room temperature. Within the $150 \mathrm{ps}$ evolution time considered, thermally stimulated configuration modifications may have a sufficiently high probability to take place. The slowing down is characterized by several significant features. These can be illustrated with the help of Figure 5 which represents in the cluster before its deposition and in the cluster-substrate system after $150 \mathrm{ps}$ evolution. For example, the $\mathrm{Co}_{285} \mathrm{Ag}_{301}$ cluster is represented and the slowing down energy is $0.5 \mathrm{eV} /$ atom. The final system is characterized by a limited penetration of the cluster into the substrate.

It undergoes some deformation accompanied by structural accommodation of the cluster with the substrate, which is limited in the case of Figure 5. At the same time, the upper part of the cluster may retain its initial atomic arrangement. In the case of Figure 5, some damage is created in the substrate and the Ag cluster shell tends to spread on the substrate surface. While the Co core is only moderately affected by the slowing down, the Ag lattice, which is already distorted initially, undergoes further deformation as a consequence of the impact.

In Figure 6 the film grown by deposition of $0.5 \mathrm{eV} /$ atom $\mathrm{Ag}_{\mathrm{n}} \mathrm{Co}_{\mathrm{m}}$ clusters with number of atoms 200, 500,1000, 1500, 2000, 2500 and 3000 is presented after deposition of 65 clusters. From Figure 6 it is seen that some clusters are strongly deformed in result of thin film growth. Core-shell clusters -depending on the binding energy of the core may display defects resulting from the competition between epitaxial accommodation and the random core orientation. The lattice in the free clusters is distorted and this distortion is enhanced by the impact. The memory of the initial cluster morphology is partially preserved after the impact 

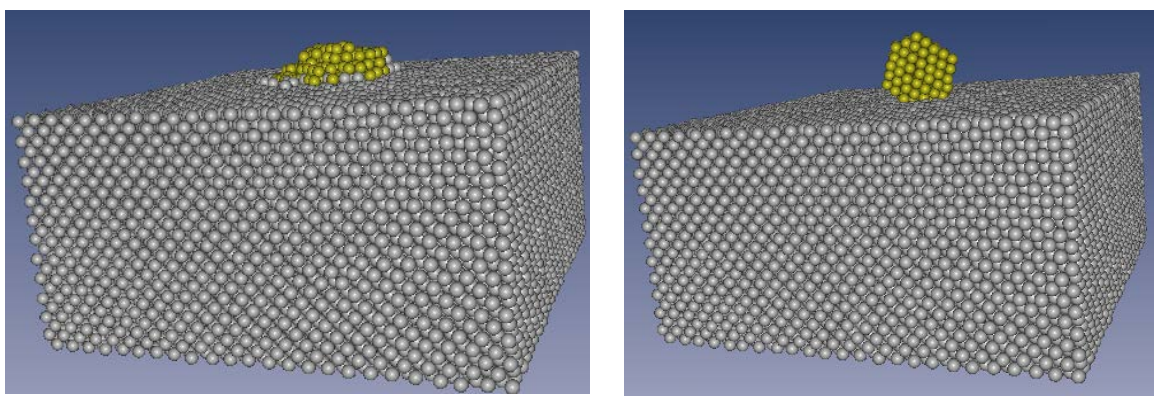

Figure 5. Initial and final configuration of a $\mathrm{Co}_{10} \mathrm{Ag}_{191}$ cluster slowing down at 0.5 eV/atom kinetic energy.

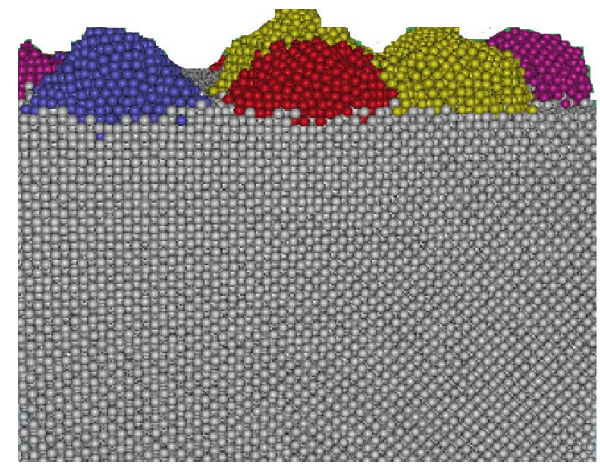

Figure 6. Thin film growth by low energy cluster deposition.

of the larger cluster, both as the core and the shell are concerned. No such memory effect was found for the small cluster, which morphology is destroyed by the impact and its reshaping is governed by the epitaxial accommodation. This process is only partial for the larger cluster considered, within the simulation time of 150 ps. Hence, at the atomic level, the cluster-substrate system displays a complex structure after deposition and the memory of the initial geometrical properties is only partial. This may have consequences relevant to catalysis, magnetic and optical properties and further work is in progress to gather a better insight about the possibilities to monitor the consequences of the cluster-surface interaction.

Obtained results show that the parallelization in Massage Passing of the classical MD code with EAM potentials gives good results on a cluster of personal computers.

\section{Conclusions}

1) The results of this research program illustrated how classical cluster modeling can contribute to understanding the properties of nanoparticles and the nanostructured materials formed by them.

2) Large-scale computations are proposed for modeling nanostructured Ag-Co materials synthesized by cluster deposition. For this, molecular dynamic codes have been developed for working on parallel computers. 
3) It is shown that the damage of the substrate is significant and depends on the deceleration energy. This can lead to the fact that the Ag step surrounds the cluster, which can have a height of more than one atomic layer, and individual additional atoms or small islands of the monolayer, except for the step. The latter comes from the cluster, and the former from the substrate.

4) Information is received on the effects of exposure to the penetration of a cluster into a crystal, its deformation and lattice distortions, with an emphasis on cluster size and stoichiometry.

\section{Acknowledgements}

One of the authors (A. Rasulov) thanks the professor of the Universite Libre de Bruxelles A. Dzhurakhalov for critical comments and useful advice in discussing the results of this work.

\section{Conflicts of Interest}

The authors declare no conflicts of interest regarding the publication of this paper.

\section{References}

[1] Dzhurakhalov, A., Rasulov, A., Van Hoof, T. and Hou, M. (2004) Ag-Co Clusters Deposition on $\mathrm{Ag}(100)$ : An Atomic Scale Study. European Physical Journal, D31, 53-61. https://doi.org/10.1140/epjd/e2004-00115-2

https://www.researchgate.net/publication/225716851 Ag-Co clusters deposition o n Ag100 An atomic scale study https://link.springer.com/article/10.1140/epjd/e2004-00115-2

[2] Rasulov, A.M. (2016) Kompyuternoe modelirovanie protsessov rasseyaniya i implantatsii ionov v tonkikh monokristallakh. Printing Center of Science and Technology.

http://isbn.natlib.uz/uz-Latn-UZ/Search/Search?page=1538\&size=10\&type=1

[3] Rasulov, A.M., Umarov, F.F., Dzhurakhalov, A.A. and Sagyndykov, A.B. (2014) Computer Simulation of Low-Energy Ion Near-Surface Implantation at Channeling Conditions and Different Mass Ratio of Colliding Particles. ICACS-26. Debrecen, Hungary, 208. https://agenda.infn.it/event/7409/contributions/67677/contribution.pdf

[4] Hou, M., Kharlamov, V.S. and Zhurkin, E.E. (2002) Atomic-Scale Modeling of Cluster-Assembled $\mathrm{Ni}_{\mathrm{x}} \mathrm{Al}_{1-\mathrm{x}}$ Thin Films. Physical Review B, 66, 195408-1.

https://www.researchgate.net/publication/292615815 Processy formirovania nano struk-

tur pri osazdenii klasterov $\mathrm{Na}$ poverhnost kristallov $\mathrm{S}$ pomosu komputernogo modelirovania https://doi.org/10.1103/PhysRevB.66.195408

[5] Hou, M., El Azzaoui, M., Pattyn, H., Verheyden, J., Koops, G. and Zhang, G. (2000) Growth and Lattice Dynamics of Co Nanoparticles Embedded in Ag: A Combined Molecular-Dynamics Simulation and Mössbauer Study. Physical Review B, 62, 5117. https://journals.aps.org/prb/issues/62/8 https://doi.org/10.1103/PhysRevB.62.5117

[6] Van Hoof, T. and Hou, M. (2004) Surface Effects on Structural and Thermodynam- 
ic Properties of $\mathrm{Cu}_{3} \mathrm{Au}$ Nanoclusters. Applied Surface Science, 226, 94-98.

https://www.sciencedirect.com/journal/applied-surface-science/vol/226/issue/1 https://doi.org/10.1016/j.apsusc.2003.11.036

[7] Oh, D.J. and Johnson, R.A. (1988) Simple Embedded Atom Method Model for fcc and hcp Metals. Journal of Materials Research, 3, 471-478. https://doi.org/10.1557/JMR.1988.0471

[8] Johnson, R.A. (1989) Alloy Models with the Embedded-Atom Method. Physical Review $B, 39,12554$. https://aip.scitation.org/doi/10.1063/1.4804954 https://doi.org/10.1103/PhysRevB.39.12554 